\title{
ON SYMMETRIC PRODUCTS OF CURVES
}

\section{ARTHUR MATTUCK}

In view of the increasing fashionability of symmetric products these days, we would like to note here that certain proofs in the algebro-geometric literature about curves and their Jacobians gain somewhat in clarity if they are placed on the symmetric product of the curve with itself. At the same time one learns something about the symmetric products. We have in mind Weil's proof (still the most elementary one) of the Castelnuovo-Severi inequality lying at the base of the Riemann hypothesis in function fields, Matsusaka's proof of the intersection relations among the $W_{i}$ on a Jacobian, and the classical theory of Weierstrass points on a curve. In the first two cases, the proofs given here are essentially the original ones, only cleaned up a little (though we do get a slight sharpening of the inequality as a by-product); in the third case, it is a question of giving the geometric significance of some calculations whose meaning I could never understand.

1. The Castelnuovo-Severi inequality. Let $C$ be a complete nonsingular curve over an algebraically closed field $k$; we dismiss the trivial and semi-trivial cases and assume henceforth that $g \geqq 2$, where $g$ is the genus of $C$. Let $C[g]$ and $C(g)$ be the $g$-fold direct and symmetric products of $C$ with itself, respectively; these are both nonsingular varieties $[6$, p. 803] and we can think of their points as representing respectively the ordered $g$-tuples of points of $C$, and the unordered $g$-tuples (positive divisors of degree $g$ on $C$ ). Some of the important $g-1$ dimensional subvarieties of $C(g)$ are first of all the "simple coincidence locus" $\Delta_{1}$, consisting of all divisors $2 p_{1}+p_{2}+\ldots$ $+p_{o-1}$ containing a repeated point, secondly the variety $X[p]$, which consists of all divisors containing a given $p \in C$ and which is evidently isomorphic to $C(g-1)$, and finally the unique positive $g-1$ cycle $S$ in the canonical system on $C(g)$.

A few words about $S$ to begin with. Let $x_{1}, \cdots, x_{0}$ be independent generic points of $C$, and for a function $f$ of $k(C)$, denote by $f^{(\alpha)}$ the function $f\left(x_{\alpha}\right) \in k\left(x_{\alpha}\right)$, and similarly for the differentials $\phi$ of the field $k(C)$. Then if $\left\{f_{i} \phi\right\}, i=1, \cdots, g, f_{1}=1$, are a basis for the regular differentials on $C$, the $g$ simple differentials $\sum_{\alpha} f_{i}^{(\alpha)} \phi^{(\alpha)}$ are a basis for the regular 1-forms on $C(g)$ and their exterior product

$$
\Phi=\operatorname{det}\left|f_{i}^{(\alpha)}\right| \phi^{(1)} \cdots \phi^{(0)}=\operatorname{det}\left|f_{i}^{(\alpha)} \phi^{(\alpha)}\right|
$$

Received by the editors December 27, 1960. 
is a regular $g$-form on $C(g)$, and in fact up to a constant factor, the unique one. Its zero-locus is thus a positive cycle $S$. Actually, $S$ is irreducible [4], though for our later purposes it will be enough to know that all its components occur with multiplicity one, as we shall show. $S$ consists exactly of the special divisors of degree $g$ on $C$ those $g$-tuples $p_{1}+\cdots+p_{g}$ belonging to a linear system of positive dimension, or equivalently for which there exists a regular differential $\psi$ on $C$ such that $(\psi)>p_{1}+\cdots+p_{g}$. For $\Phi$ is zero at a point of $C(g)$ if and only if the rows of the determinant become linearly dependent, and this happens exactly when such a $\psi$ exists, [6].

From the definition of $S$, we have almost immediately

$$
2 S+\Delta_{1} \sim 2 \sum_{1}^{20-2} X\left[p_{i}\right]
$$

where $\sim$ denotes linear equivalence on $C(g)$ and $p_{1}+\cdots+p_{o}=(\phi)$ is a canonical divisor on $C$. Namely, $\left(\operatorname{det}\left|f_{i}^{(\alpha)}\right|\right)^{2}$ is a function on $C(g)$ whose zeros are the left side and poles the right side: this is clear set-theoretically, and the multiplicities are easily calculated-that of $S$ by definition of $S$, that of $X\left[\mathfrak{p}_{i}\right]$ trivially because the determinant is linear in each entry, and for the multiplicity of $\Delta_{1}$ one can use the local uniformizing parameter $\prod_{\alpha \neq \beta}\left(t^{(\alpha)}-t^{(\beta)}\right), t$ being any function on $C$ which is nonconstant.

Now let $\mathfrak{X}$ be a nontrivial correspondence class on $C \times C$. By an elementary argument $[4$, p. 44] it contains an irreducible correspondence $Z$ over $K \supset k$ of degrees $g$ and $e$, and such that $Z(\mathfrak{p})=q_{1}+\ldots$ $+q_{o}$ is a generic point of $C(g)$ over $k, \mathfrak{p}$ being generic over $K$. For if $Z^{\prime}$ is some correspondence in the class $\mathfrak{X}$ whose first degree $d^{\prime}$ is large and one picks fixed generic points $\mathfrak{r}_{1}, \cdots, \mathfrak{r}_{d^{\prime}-0}$ of $C$, then $Z^{\prime}(\mathfrak{p})$ $-\left(\mathfrak{r}_{1}+\cdots+\mathfrak{r}_{d^{\prime}-0}\right)$ is a divisor of degree $g$ and is therefore by the Riemann-Roch theorem equivalent to a unique positive divisor of degree $g$, which it is easy to see must contain only points which are independent generic over $k$. Set therefore $Z=Z^{\prime}-\sum C \times \mathfrak{r}_{i}$. Thus $\mathfrak{p} \rightarrow Z(\mathfrak{p})$ gives a rational map of $C \rightarrow C(\mathrm{~g})$ whose image is a curve $T$. Since $Z(p)$ is nonspecial, $S \cdot T$ is defined and is non-negative, and since clearly $\left[T \cdot X\left[\mathfrak{p}_{i}\right]\right]=e$, we get for $g \geqq 2$ and $Z$ nontrivial, by applying (1),

$$
\frac{1}{2}\left[\Delta_{1} \cdot T\right] \leqq(2 g-2) e .
$$

This is a little sharper than Castelnuovo's inequality, where the right side is $(2 g-1) e$ for all $g$ and $X$; here $\Delta_{1} \cdot T$ is what he calls the number of "coincidences" for the correspondence $Z$. 
To get the Severi form of the inequality, there is little to add to Weil's proof, but we sketch the outline for convenience. Let $D$ be the diagonal of $C \times C$ and let $f: C[g] \rightarrow C(g)$ and $\operatorname{pr}_{12}: C[g] \rightarrow C \times C$ be the usual maps. Consider the cycle $(1 /(g-2) !)\left(\operatorname{pr}_{12}^{-1} D\right) \cdot\left(f^{-1} T\right)$ on $C[g]$; applying first $f$ and then $\operatorname{pr}_{12}$ to it and using the projection formula in each case gives then $\left[\Delta_{1} \cdot T\right]=\left[D \cdot\left(Z \circ{ }^{t} Z-e D\right)\right]$. Namely, $Z \circ{ }^{\prime} Z$ is the locus over $K$ of $\sum q_{i} \times q_{j}$, while $\operatorname{pr}_{12}\left(f^{-1} T\right)$ is $(g-2)$ ! times the locus over $K$ of $\sum_{i \neq j} \mathfrak{q}_{i} \times \mathfrak{q}_{j}[4$, p. 48]; their difference is the $K$-locus of $\sum q_{i} \times q_{i}$, but as $\mathfrak{p}$ varies on $C$, this moving zero-cycle evidently covers the diagonal $D$ just $e$ times. Thus since $[D \cdot D]=2-2 g$, the inequality (2) above becomes

$$
d(Z) d\left(Z^{\prime}\right)-\frac{1}{2}\left[Z \circ{ }^{\prime} Z \cdot D\right] \geqq e .
$$

Since the left side of (3) is an invariant of the correspondence class, (3) is valid for an arbitrary nontrivial $Z$, if $g \geqq 2, e$ being then $d^{\prime}\left(Z_{0}\right)$ where $Z_{0}$ is an irreducible correspondence of degree $g$ in the class of $Z$. For example, if $g \geqq 2$, then $e=1$ only if $Z_{0}$ defines a rational inseparable map $C \rightarrow C$, in which case $Z_{0} \circ{ }^{t} Z_{0}=g D$. Thus in all cases, if $g \geqq 2$, $Z$ nontrivial, then (3) holds with $e=2$.

2. Intersection relations on Jacobians. Let $J$ be the Jacobian, $\pi: C(g) \rightarrow J$ the map with reference point $\mathfrak{p} \in C$. We have then the usual subvarieties (indexed by codimension) $\Theta=W_{1}, \cdots, W_{0}$, and we want to show that, modulo numerical equivalence,

$$
W_{r} \cdot W_{1} \equiv(r+1) W_{r+1} \text {. }
$$

Now on $C(g)$ we have the subvarieties: $X_{r}=\{$ all divisors containing $r p\}$, and $\Delta_{r+1}=\{$ all divisors of the form $r p+2 q+\cdots\} . \Delta_{r+1}$ is so to speak the simple coincidence locus on $X_{r}$. We have then by definition $\pi\left(X_{r}\right)=W_{r}$, and the formulas (cf. [4])

$$
\begin{aligned}
\pi^{-1}\left(W_{1}\right) & =S+X_{1}, \\
X_{r} \cdot X_{1} & \sim X_{r+1}, \\
X_{r} \cdot \Delta_{1} & \equiv 2 r X_{r+1}+\Delta_{r+1} .
\end{aligned}
$$

Here (6) is meant in the sense of the rational equivalence ring on $C(g)$, since of course $X_{r} \cdot X_{1}$ is not defined; in (7) numerical equivalence is meant. The second and third of these are essentially trivial: it is just the old game of turning obvious intersection multiplicities on $C[g]$ into less obvious ones on $C(g)$ by applying the projection formula to the map $f$.

To prove (5), we note first that it is true set-theoretically, since $\pi\left(\sum \mathfrak{p}_{i}\right) \in W_{1}$ if and only if $\sum \mathfrak{p}_{i} \sim \mathfrak{b}$, where $\mathfrak{b}=\mathfrak{p}+q_{1}+\cdots+q_{b_{-1}}$ is a 
divisor lying in $X_{1}$. That is, $\sum \mathfrak{p}_{i}-\mathfrak{p} \sim \sum \mathfrak{q}_{i}>0$, which happens if and only if $\mathfrak{p}_{i}=\mathfrak{p}$ for some $i$ (so that $\sum \mathfrak{p}_{i} \in X_{1}$ ), or if $\sum \mathfrak{p}_{i}$ is special (so that $\left.\sum p_{i} \in S\right)$. Thus in any case, $\pi^{-1}\left(W_{1}\right)=S^{\prime}+a X_{1}$, where $a \geqq 1$ and $S^{\prime}$ is a cycle with the same support as $S$. By applying $\pi$ and noticing that $\pi\left(S^{\prime}\right)=0$, we get $a=1$. Now letting $X^{1}$ be the curve on $C(g)$ consisting of all divisors of the form $q+q_{1}+\cdots+q_{0-1}$ where the $q_{i}$ are fixed points, then for general choice of the $q_{i}$, we get

$$
\pi^{-1}\left(W_{1}\right) \cdot X^{1}=S^{\prime} \cdot X^{1}+X_{1} \cdot X^{1} \text {. }
$$

Using $\left[W_{1} \cdot \pi\left(X^{1}\right)\right]=g$ (cf. [2]) and (6) above, we get

$$
\left[S^{\prime} \cdot X^{1}\right]=g-1
$$

while from (1), (6), and (7), we have

$$
\left[S \cdot X^{1}\right]=g-1 .
$$

Now set-theoretically, $|S| \cap X^{1}$ contains in general $g-1$ points, since there are just $g-1$ ways of completing $q_{1}+\cdots+q_{o-1}$ to be a special divisor of degree $g$-just pick any one of the $g-1$ additional points which fill out the in general unique canonical divisor of degree $2 g-2$ containing $q_{1}+\cdots+q_{b-1}$. And every component of $S$ is intersected properly by some suitable $X^{1}$. It follows that all the components of $S$ and $S^{\prime}$ must occur with multiplicity one, so that from $|S|=\left|S^{\prime}\right|$, we deduce $S=S^{\prime}$, which proves (5).

We can now prove the relations (4). Using in turn the projection formula, (1), (5), (6), and (7), we get

$$
W_{r} \cdot W_{1}=\pi\left(X_{r} \cdot\left(S+X_{1}\right)\right) \equiv(2 g-1-r) W_{r+1}-\frac{1}{2} \pi\left(\Delta_{r+1}\right) .
$$

And just as in Matsusaka, we get to complete the proof $(*=$ Pontrjagin product)

$$
\pi\left(\Delta_{r+1}\right) \equiv W_{r+2} *(2 \delta)\left(W_{o-1}\right) \equiv W_{r+2} * 4 W_{o-1}=4(g-r-1) W_{r+1} .
$$

The only nontrivial part of these last steps, and the only place in this proof where numerical equivalence enters essentially (all the rest could be done with rational equivalence) is in the statement $(2 \delta)\left(W_{0-1}\right)$ $\equiv 4 W_{o-1}$. We give Matsusaka's proof: put $\alpha=n \delta, W=W_{o-1}$, and let us show that $\alpha(W) \equiv n^{2} W$. For any positive divisor $X$, we have (since it is a question of zero-cycles), on the one hand $\alpha^{-1}(\alpha(W) \cdot X)$ $\equiv \nu(\alpha)(\alpha(W) \cdot X)$ and on the other hand $\alpha^{-1}(\alpha(W) \cdot X)=\alpha^{-1}(\alpha(W))$ $\cdot \alpha^{-1}(X) \equiv \nu(\alpha) W \cdot n^{2} X$ by the theorem of the square and since $\alpha^{-1}(\alpha(W))$ consists of the $\nu(\alpha)$ translates of $W$ by the elements of the kernel of $\alpha$.

3. Weierstrass points. These are by definition the points $p \in C$ for 
which $g \mathfrak{p}$ is a special divisor, and are thus in one-one correspondence with the points of $S \cap \Delta$, where $\Delta$ is the curve on $C(g)$ which represents all divisors of form $g \mathfrak{p}$. The classical theorem is that if $N=$ the number of Weierstrass points, then in characteristic zero,

$$
2(g+1) \leqq N \leqq g^{3}-g
$$

with the lower equality holding if and only if $C$ is hyperelliptic. This is proved by defining a weight $w(p)$ for $\mathfrak{p} \in C$, which is a non-negative integer, positive exactly when $\mathfrak{p}$ is a Weierstrass point, and showing that $w(\mathfrak{p}) \leqq g(g-1) / 2$, and that $\sum w(\mathfrak{p})=g^{3}-g$. We wish to give here the geometric meaning of this proof.

Assume $g>0$, and for $\mathfrak{p} \in C$, let $\left\{u_{i}\right\}$ be the sequence of "missing ordinals" associated with $\mathfrak{p}$ : the sequence of integers such that there is no function $f \in k(C)$ with a single pole of order $u_{i}$ at $\mathfrak{p}$. From the Riemann-Roch theorem, this sequence contains $g$ numbers, of which the first is 1 , and the last $\leqq 2 g-1$, and it is written (cf. [1] for this entire section)

$$
1, \alpha_{1}+1, \alpha_{1}+\alpha_{2}+1, \cdots, \sum_{1}^{0-1} \alpha_{i}+1
$$

Definition. The weight $w(\mathfrak{p}) \equiv(g-1)\left(\alpha_{1}-1\right)+(g-2)\left(\alpha_{2}-1\right)+\ldots$ $+\left(\alpha_{g-1}-1\right)$. Clearly $w(p)=0$ if and only if $p$ is not a Weierstrass point. Note also that the complementary sequence to $\left\{u_{i}\right\}$ is the set of occurring ordinals; these clearly form an additive semigroup. Now if 2 is an occurring ordinal, $C$ is clearly hyperelliptic, and in this case (characteristic zero), a direct calculation shows that all Weierstrass points must have the sequence $1,3,5, \cdots, 2 g-1$ and thus weight $g(g-1) / 2$. Otherwise,

Proposition 1. If $C$ is not hyperelliptic, $w(\mathfrak{p})<g(g-1) / 2$.

Proof. The sequence for any Weierstrass point must then start $1,2, \cdots$ and end with a number $\leqq 2 g-1$, contain $g$ members, and have its complementary sequence a semigroup. The proposition follows formally from these facts by induction on $g$. For $g=3$, there are only two possible sequences and they both have the right weight. Assuming the truth for $g$, and considering it for $g+1$, we thus have a sequence $1,2, \cdots, \sum \alpha_{i}+1$. It cannot end with the numbers $2 g$, $2 g+1$, for then the complementary sequence would not have the semigroup property: one cannot choose $g$ numbers from $3, \cdots, 2 g-1$ such that no two add up to either $2 g$ or $2 g+1$. Therefore the sequence ends with at most one of these two numbers. Drop the last term of the sequence; one is left then with a $g$-membered sequence satisfying the induction hypothesis, of weight $<g(g-1) / 2$, and it is immediate 
that then the weight of the original sequence cannot be more than $g$ bigger, is therefore $<g(g+1) / 2$.

PROPOSITION 2. In characteristic zero, $w(p)$ is the multiplicity of $g p$ in $\Delta \cdot S$.

Proof. Let $t \in k(C)$ be a local uniformizing parameter at $\mathfrak{p}$, and put $t_{\alpha}=t\left(x_{\alpha}\right)$. By $\$ 1$, $\left(\operatorname{det}\left|f_{i}\left(t_{\alpha}\right)\right|\right)^{2} / \Pi\left(t_{\alpha}-t_{\beta}\right)$ is a local function for the cycle $2 S$ in the neighborhood of $g \mathfrak{p}$ on $C(g)$. Its restriction to $\Delta$ is by an elementary calculation just $W\left(f_{1}(t), \cdots, f_{o}(t)\right)^{2}$, the square of the Wronskian, which doesn't vanish identically since the $f_{i}(t)$ are independent on $C$ (characteristic zero!). The multiplicity we seek is therefore $\operatorname{ord}_{\mathfrak{p}} W$, and this is computed as follows. Recall that the $f_{i}$ are any basis for the space of functions $f$ on $C$ such that $(f)$ $\geqq-(\phi)$. By Riemann-Roch, if $u_{i}$ is a missing ordinal, then there is a function $f_{i}$ in the space with a zero of order $u_{i}-1$ at $\mathfrak{p}$, so that $f_{i}=t^{u_{i}-1}+\cdots$. These $f_{i}$ are $g$ in number, clearly are independent, are therefore a basis for the vector space, and using them to compute $\operatorname{ord}_{\mathfrak{p}} W$, one gets $\left(\right.$ since $\left.\left(u_{i}-1\right)=\alpha_{1}+\cdots+\alpha_{i-1}\right)$

$$
\operatorname{ord}_{\mathfrak{p}} W=\sum_{1}^{g}\left(u_{i}-1\right)-\frac{g(g-1)}{2}=w(\mathfrak{p}) .
$$

It follows that $\sum w(\mathfrak{p})=[\Delta \cdot S]$ in characteristic zero. To complete now, we use the first two sections to prove

Proposition 3. In any characteristic, $[\Delta \cdot S]=g^{3}-g$, if defined.

Proof. From the end of $\S 2$, we have $\pi(\Delta)=(g \delta) W_{g-1} \equiv g^{2} W_{g-1}$. Also, we have $\left[\Delta \cdot X_{1}\right]=g$, by a proof like that of (6) and (7). By (5), we have $\Delta \cdot \pi^{-1}(W)=\Delta \cdot\left(S+X_{1}\right)$, and applying the projection formula to this gives

$$
g^{2}\left[W_{g-1} \cdot W\right]=[\Delta \cdot S]+\left[\Delta \cdot X_{1}\right],
$$

from which the result follows, using (4).

\section{BiBLIOGRAPHY}

1. Hensel-Landsberg, Theorie der algebraischen Funktionen einer Variabeln ...., Leipzig, Teubner, 1902.

2. S. Lang, Abelian varieties, New York, Interscience, 1959.

3. T. Matsusaka, On a characterization of a Jacobian variety, Mem. Coll. Sci. Univ. Kyoto. Ser. A. Math. vol. 23 (1959) pp. 1-19.

4. A. Mattuck, Symmetric products and Jacobians, Amer. J. Math. vol. 83 (1961) pp. 189-206.

5. A. Weil, Sur les courbes algébriques et les variêtés qui s'en déduisent, Paris, Hermann, 1948.

6. A. Andreotti, On a theorem of Torelli, Amer. J. Math. vol. 80 (1958) pp. 801-828.

\section{Massachusetts Institute of Technology}

